\title{
Compliance and persistence with osteoporosis medications: A critical review of the literature
}

\author{
Stuart Silverman • Deborah T. Gold
}

Published online: 5 May 2010

(C) The Author(s) 2010. This article is published with open access at Springerlink.com

\begin{abstract}
It is widely acknowledged that compliance and persistence with oral osteoporosis medications, particularly with bisphosphonates, is poor. Several excellent reviews have been written on compliance and persistence with osteoporosis medications and have discussed improvements seen with extended dosing intervals. This review begins with studies on extended dosing intervals to examine the limitations of administrative claims data. It also looks at compliance and persistence across multiple medical conditions, examining the importance of prescription fulfillment, intentional choice, causation and possible interventions.
\end{abstract}

Keywords Osteoporosis · Persistence $\cdot$ Compliance $\cdot$

Adherence

\section{The negative consequences of poor adherence}

Using claims databases several authors have found an association between compliance and persistence with osteoporosis medication and fracture risk reduction. Siris in 2006 [1] examined the relationship of persistence and medical possession ratio (MPR) to fracture rate of vertebral and nonvertebral fractures. Total vertebral, nonvertebral and hip fractures were significantly lower in compliant and

\section{S. Silverman $(\triangle)$}

Cedars-Sinai/UCLA,

8641 Wilshire Blvd., Suite 301,

Beverly Hills, CA 90211, USA

e-mail: stuarts@omcresearch.org

\section{T. Gold}

Duke University Medical Center, Box 3003, Durham, NC 27710, USA

e-mail: dtg@geri.duke.edu persistent patients with relative risk reductions of $20-45 \%$. Huybrechts [2] studied the relationship between compliance as measured by medication possession ratio (MPR) and fracture rate in a US managed care database. Low compliance as measured by a MPR of $<80 \%$ was associated with a $17 \%$ increase in fracture rate, adjusting for other known risk factors. Low compliance was also associated with a $37 \%$ increase in risk of all cause hospitalization and average monthly costs of all medical services were higher. Rabenda [3] used the Belgian national security database to study the relationship of MPR to hip fracture risk. For each decrease of $1 \%$ in MPR, the risk of hip fracture increased by $0.4 \%$. The relative risk reduction at 12 months for hip fracture was $60 \%$ for persistent compared to nonpersistent patients.

\section{Adherence and risk of fracture: is there a healthy adherer bias?}

The positive effects of adherence on fracture risk reduction noted above may also arise from what has been termed "healthy adherer bias". Adherence maybe a marker for adherence to effective treatments other than study medications or to other adherence behaviors that effect outcomes. Granger [4] in the CHARM trial found that good adherence was associated with lower all cause mortality in both treatments and placebo arms. If patients who adhere to osteoporosis pharmacologic therapies are more likely to take calcium, vitamin D, and engage in physical activity, for example, then the projected effects of calcium, vitamin $\mathrm{D}$, and physical activity maybe falsely attributed to the pharmacologic therapy. However, there have been no studies to date that have clearly addressed this issue in the osteoporosis literature. 
One recent study found no evidence of healthy adherer bias in explaining survival after acute myocardial infarction with adherence to medications improving mortality benefits such as statins and beta-blockers but no calcium channel blockers being associated with increased survival [5]. However, these data are limited in that they refer to secondary prevention rather than primary prevention. Secondary prevention indicates a negative health event, such as heart attack or fracture, has occurred prior to patients adhering to therapy after a cardiac event or fracture. In such cases, the event may drive patients to engage in protective behaviors to avoid additional future events. However, it is not likely that healthy adherer bias will play a major role in osteoporosis. The epidemic of vitamin D deficiency is illustrated by Holick [6] who reports that over $50 \%$ of postmenopausal women on osteoporosis therapies do not have adequate vitamin $\mathrm{D}$ levels.

A second type of healthy adherer bias has been pointed out is that patients who are well adherent to placebo in drug trials also have significant fracture reduction effect. This has been pointed out by Curtis [7] in an analysis of Women's Health Initiative (WHI). Examining adherence to medication in the placebo arm of the estrogen/hormone therapy component of the WHI $(n=13,485)$, Curtis [7] found that there was a consistent association between adherence to placebo and protective effect for all outcomes that was especially strong for hip fracture and death. They conclude that medication adherence which can be measured in administrative databases or other secondary data sources maybe a proxy for other positive health behaviors (e.g., adequate calcium and vitamin D intake, exercise) that may not be available in these databases.

\section{Adherence and dosing interval: do longer dosing intervals improve compliance and persistence?}

Data from both administrative claims data and pharmacy claims data have shown improved persistence and compliance (as measured by medication possession ratio) on oral bisphosphonate therapy with extended dosing intervals from daily to weekly. Data on compliance and persistence with monthly bisphosphonate therapy have been equivocal. Several studies have shown improved compliance with a longer dosing interval, while others have not. Several factors may contribute to these findings: the limitations of pharmacy claims data which do not allow for correction due to confounding variables which administrative claims do, or methodologic definitions of the refill gap (the length of the time between doses that is allowed to define persistence on therapy). Monthly ibandronate for example showed improved persistence when the refill gap was measured as
45 days as compared to 30 days, the standard refill gap for weekly bisphosphonate therapy showed improved persistence [8]. When the refill gap was defined as 30 days for both, then there was no superiority for monthly ibandronate. Gold and colleagues [9] using pharmacy claims data and a 90-day refill gap did not find improved persistence with monthly over weekly therapy. They report that they used $15,30,45,60$, and 90 day refill gaps without finding any meaningful differences in outcomes.

\section{Limitations of claims data}

The studies mentioned above which use administrative claims data have limitations due to the nature of such data. Claims data do not tell us about samples given to patients or whether patients fulfill their scripts (primary medication nonadherence, see below). Claims data provide information on procedures and visits but may not include key control variables. For example important variables in any study of osteoporosis patients are likely to be bone density (DXA) measurements or calcium intake, but typically this information is not available in a claims database. Because DXA is a procedure, it is possible to determine that the test was reimbursed but not what its outcomes are. Further, in observational studies, outcomes can reflect a lack of comparability between treatment groups rather than the effects of treatment. Propensity scores show promise in helping to adjust for such selection bias [10].

\section{Compliance with medications}

There are multiple studies that have shown poor compliance with dosing instructions with use of oral bisphosphonates for the treatment of osteoporosis. Hamilton and colleagues [11] found that 57/219 patients on risedronate did not follow instructions properly. Those who stayed upright after taking the medicine were more likely to have adverse events and discontinue. Approximately one-third of patients who experienced adverse events were rechallenged with the drug, and almost $50 \%$ were able to restart. This study makes clear that having a specialized nurse or other healthcare professional follow up each long-term prescription with feedback to the patient improves clientprofessional relationships.

\section{Primary medication nonadherence}

Nonadherence has two levels: primary and secondary. We know very little about primary medication nonadherence in osteoporosis and are only beginning to understand the 
dimensions of primary nonadherence in other disease states. While early compliance and persistence data were developed from self-report [12] with the advent of e-prescribing, prescriptions written can be matched to prescriptions filled [13].

The first studies collected information using self-report. Kennedy [12] used data from the 2004 Medicare current beneficiary survey, an ongoing national panel survey conducted by the Center for Medicare and Medicaid Services, to study possible patterns in unfilled Medicare prescriptions. The sample included 14,464 communitydwelling Medicare beneficiaries who were asked if there were any prescriptions they had not filled that were written or phoned in by their physician. Respondents were asked to answer about medications in general as they were not asked the names or types of specific medications. In 2004, an estimated 1.6 million Medicare beneficiaries or $4.4 \%$ admitted failing to fill or refill at least one prescription. The most common reasons for not filling these prescriptions included the following: (1) cost $(55.5 \%)$, (2) lack of insurance coverage $(20.2 \%)$, (3) did not believe medicine was necessary for their condition (18.0\%), and (4) feared medicine contraindications or reactions (11.8\%). Rates were higher in those under 65 than those 65 and older (10.4\% versus $3.3 \% ; P<0.001)$ and for women than men (5.0\% versus $3.6 \% ; P=0.001)$, for non-whites and whites (5.5\% versus $4.2 \% ; P=0.010)$, and for dually eligible Medicaid beneficiaries than for those not covered by Medicaid (6.3\% versus $4.0 \% ; P=0.001)$.

Failure to fill rates in this study were significantly higher among beneficiaries with low incomes $(P<0.001)$, psychiatric conditions $(P<0.001)$, arthritis $(P<0.001)$, cardiovascular disease $(P=0.003)$, emphysema, asthma or chronic obstructive pulmonary disease $(P<0.001)$. Rates were higher for those with more self-reported chronic conditions $(P<0.001)$. Among the prescriptions not filled, CNS agents were the most frequent identified overall, with nonsteroidals $(23.6 \%)$, followed by cardiovascular agents $(18.3 \%)$, and endocrine metabolic agents $(6.5 \%)$. The authors concluded that most Medicare beneficiaries do fill their prescriptions, though fulfillment is not $100 \%$ (Kennedy [12]).

Fischer [13] recently reported primary medication nonadherence in 75,589 patients treated in a community-based e-prescribing initiative. With 195,930 e-prescriptions, 78\% or 151,837 were filled. Of 82,245 e-prescriptions for new medications, $72 \%$ or 58,984 were filled. Primary adherence rates were higher for prescriptions written by primary care specialists/especially pediatricians $-84 \%$. Patients age 18 and younger filled prescriptions at the highest rate at $87 \%$. Medication class using multivariate analysis is the strongest predictor of adherence, and nonadherence was common for newly prescribed medicines for chronic conditions such as hypertension (28.4\%), hyperlipidemia (28.2\%), and diabe- tes $(31.4 \%)$. We do not currently have such data about osteoporosis medications. However, these findings strongly suggest that in the future, efforts to understand and improve primary adherence in osteoporosis will most likely be needed as well.

6.1 What are reasons for poor compliance and persistence?

While earlier research on compliance and persistence with osteoporosis medications focused primarily on convenience and dosing interval of oral medications, more recent studies have looked at the role of choice in poor compliance and persistence.

As pointed out by the 2002 Harris survey done by the Boston Consulting Group [14], adherence across multiple medications may often be intentional or deliberate. Recently Rees and colleagues [15] studied intentional and unintentional nonadherence to ocular or hypertensive treatment in patients with glaucoma. Overall, 59 participants (45\%) reported some degree of nonadherence, of which 39 participants $(66.1 \%)$ reported unintentional nonadherence. However, 10 (16.9\%) reported intentional nonadherence and $10(16.9 \%)$ reported both. Non-adherers were significantly younger and less likely to have other nonocular health conditions or used medicine other than their eye drops and reported lower belief in the necessary of eye drops for glaucoma. The degree of unintentional adherence was associated with lower belief in the necessary of eye drops while the degree of intentional nonadherence was associated with concerns about eye drops. The authors concluded that both intentional and unintentional dimensions are poorly understood and need additional research.

Intentional non-adherers have also been studied using the necessity concerns framework and beliefs in medicine questionnaire by Clifford [16]. Intentional non-adherers compared to adherers have lower perception of the necessity of new medications and higher level of concerns about taking them. Adversely, unintentional non-adherers were not significantly different from adherers. The conclusion is that, when patients start a new medicine for a chronic condition, intentional non-adherers hold beliefs significantly different than those of adherers and unintentional non-adherers.

\section{Physician and patient attitudes}

Additional causes of poor compliance and persistence with medication may focus on the importance of communication and physician and patient health beliefs and attitudes. The patient/physician dyad has been a neglected factor in explaining why patients may not take their medications correctly. Patient and physician beliefs about medication 
maybe dissimilar but may also be an important predictor of patient outcomes. On the other hand, patient and physician perspectives maybe different. For example, in osteoporosis, patients are more concerned about potential loss of independence from fractures, while physicians maybe more concerned with reducing future fractures.

Christensen [17] studied the extent to which the patient and provider symmetry in health of locus of control beliefs was associated with adherence in patients with diabetes and hypertension. Physician and patient dyads holding similar beliefs regarding the degree of personal control that the patients have over health outcomes showed lower diastolic blood pressure $(p=0.02)$ and greater adherence $(p=0.03)$ than the dyads in which patients held stronger beliefs in their own personal control than did their physicians. These data suggest that attitudinal symmetry and locus of control may play more important roles in adherence than was previously thought.

Qualitative interviews with patients in Africa receiving antiretroviral therapy by Gusdal [18] confirmed that two important drivers were costs of medications and transportation to appointments and follow-ups, with a second driver being personal responsibility for treatment, trust in the effects of the medications, and trust in the quality of counseling. The article confirms the importance of health beliefs.

7.1 Causes of poor compliance and persistence: do race and ethnicity play a role?

While adherence to medications is suboptimal, what is not known is whether there are racial and ethnic differences in adherence. Trinacty [19] studied diabetic medication adherence in African Americans. Black patients are as likely as whites to initiate oral therapy and fill their first prescription. They have higher rates of medication discontinuation and are less adherence over time. These increased over the first 6 months but then stabilized thereafter.

\subsection{Causes of poor compliance and persistence: is it} important that osteoporosis is an asymptomatic disease?

The literature has often cited the asymptomatic nature of osteoporosis until fracture as a major cause of poor compliance and adherence. However, even in symptomatic conditions such as diabetes, adherence to oral hypoglycemic therapy ranges from $36 \%$ to $93 \%$ in patients who had treatment for 6-24 months. (Cramer [20]) Similar to diabetes, there is a low rate of treatment adherence to medication in patients with an inflammatory painful arthritis such as rheumatoid arthritis with $33-40 \%$ of patients not taking their medications as prescribed (van den Bemt [21]).
7.3 Causes of poor compliance and persistence: the role of depression

Issues around poor compliance and persistence in the face of major depression are doubled because patients must first be compliant with their medication for depression, something that is infrequent, especially after psychiatric hospitalization (Zivin [22]). The relationship between osteoporosis and depression is complex, with depression potentially being both a risk factor and a consequence of fractures (Cizza [23]). As a result, the impact of depression on compliance and persistence with osteoporosis medication is complex as well. In a secondary analysis of data from the Fracture Intervention Trial (FIT), Buist [24] found that depression was a significant predictor of early discontinuation of medication - in this case, alendronate. Compliance and persistence with osteoporosis medications in the face of depression is an area that needs additional research.

7.4 Causes of poor compliance and persistence: the role of alcohol

In an early study of alcohol consumption and its impact on compliance with type II diabetes recommendations, Johnson [25] found that drinkers were significantly less likely to comply with dietary recommendation $(p=0.01)$, exercise $(p<0.05)$ oral medication use $(p<0.01)$, and attending follow-up clinic visits $(p<0.05)$ (Johnson). Some years later, alcohol misuse as measured by a brief screening questionnaire was found to be associated with an increased risk for medication nonadherence to oral antihypertensives and hyperlipidemic agents but not for oral hypoglycemics. However, oral hypoglycemic users had a higher proportion of non-drinkers which may have influenced results (Bryson [26]).

7.5 Causes of poor compliance and persistence: what is the role of switching medications?

It is not uncommon for patients on weekly bisphosphonates to be switched from 1 weekly bisphosphonate or 1 monthly bisphosphonate to another. Switching is often either based on insurance coverage or patient intolerability. In a retrospective data base analysis of pharmacy claims data, Thiebaud [27] studied a sample of 38,866 new statin users between the ages of 18 and 65 . They found that patients who were switched to another statin were significantly less compliant with medication than were those patients who remained on the same statin $(p<0.001)$; they were also less persistent by $21-48 \%(p<0.001)$ than were non-switchers. Although no similar study of osteoporosis medications has been published of which we are aware, no evidence 
suggests that the same behavior patterns would not exist in an osteoporotic population (Thiebaud [27]).

\subsection{How can we improve compliance and persistence?}

Haynes [28] published a review in the Cochrane database on how to enhance medication adherence. They searched peer-reviewed articles that reported the success of multiple randomized controlled interventions to improve medication adherence. The authors noted that 4 out of 10 articles reporting short-term treatments showed an effect on bone adherence and at least one clinical outcome. One intervention reported $1 \mathrm{RCT}$ that significantly improved patient adherence but did not enhance any clinical outcomes. For long-term treatment, 36/81 interventions from 69 RCTs were associated with significant improvement in adherence but only 25 led to improvement in at least 1 treatment outcome. Almost all of the interventions that were effective for long-term care were complex and included combining one or more of the following: community care, information reminders, self-monitoring, reinforcement, constant family therapy, psychological therapy, crisis intervention, manual telephone follow up and supportive care. Even the most effective interventions did not lead to large improvements in treatment outcomes and adherence. For short-term treatments, several quite simple interventions increased adherence. However, these interventions were inconsistent, with fewer than half the studies showing gain.

However, improvement in medication adherence has been shown in several recent studies. Conn [29] completed a meta-analysis of randomized controlled trials of older adults to determine if there were positive changes in their medication adherence. Their findings were generally positive, and the interventions most successful when a moderate proportion of participants were women, on 3-5 drugs, and when they measured pill count adherence. More specifically, Russell [30] described a clinical nurse specialist led intervention with renal transplant patients to improve adherence with immunosuppressant medications, the number 1 predictor of poor outcomes including death. Continuous self-improvement feedback, a theory-based systems approach, plus an objective marker of compliance (electronic medicine cap) led patients to significantly improve their adherence.

Unfortunately, staff intensive interventions can be expensive, so the challenge is to find cost effective ways to motivate patients both for fulfillment of the initial prescription and for persistence over time. Schackman [31] explored the feasibility of using audio computerassisted self interview techniques in routine HIV care. The intervention helped identify patients at risk for poor adherence but did not improve clinical outcomes
Accommodating patient goals and preferences in shared decision-making improves adherence and outcomes in poorly controlled asthma. Using the shared decision making process with 612 adults with poorly controlled asthma resulted in significantly better controller adherence $(p<$ $0.0001)$ as well as significantly better clinical outcomes (asthma-related quality of life, health care use, rescue medication use, asthma control, and lung function) (Wilson [32]). From these data, the authors suggest that shared decision-making where clinicians and patients negotiate a treatment regimen that accommodates patient's goals and preferences significantly improves adherence and outcomes in asthma patients. This approach has not been tested with osteoporosis patients.

\section{Conclusions}

Although we recognize the importance of compliance and persistence with osteoporosis medications improving fracture outcomes, we are only beginning to study the multiple causes of poor compliance and persistence. Until recently, simple forgetfulness was blamed for poor medication behavior. Several researchers have shown that forgetfulness is only one small reason that individuals do not take their medicines as directed. Intentional choice of noncompliance and differences between patient and physician attitudes play larger roles in this potentially harmful patient behavior. Ultimately, the most effective interventions to change this behavior will likely be behavioral interventions to improve compliance and persistence that are cost effective and scalable.

Open Access This article is distributed under the terms of the Creative Commons Attribution Noncommercial License which permits any noncommercial use, distribution, and reproduction in any medium, provided the original author(s) and source are credited.

\section{References}

1. Siris ES, Harris ST, Rosen CJ, et al. Adherence to bisphosphonate therapy and fracture rates in osteoporotic women: relationship to vertebral and nonvertebral fractures from 2 US claims databases. Mayo Clin Proc. 2006;81:1013-22.

2. Huybrechts KF, Ishak KJ, Caro JJ. Assessment of compliance with osteoporosis treatment and its consequences in a managed care population. Bone. 2006;38:922-8.

3. Rabenda V, Mertens R, Fabri V, Vanoverloop J, Sumkay F, Vannecke C, et al. Adherence to bisphosphonates therapy and hip fracture risk in osteoporotic women. Osteoporos Int. 2008; 19:811-8.

4. Granger BB, Swedberg K, Ekman I, et al. Adherence to candesartan and placebo and outcomes in chronic renal failure in the CHARM program, double-blind randomized controlled trial. Lancet. 2005;366:2005-11. 
5. Rasmussen JIN, Chong A, Alter DA. 2007 Relationship between adherence to evidence-based pharmacotherapy and long term mortality of people with myocardial infarctions. JAMA. 2007;297 (2):177-86.

6. Holick MF. Vitamin D deficiency. N Engl J Med. 2007;357:26681.

7. Curtis J, Larson J, Delzell E, Judd S, Safford MM, LaCroix A, et al. Does the benefit of medication adherence relate more to a drug effect or the behavior Itself? Quantifying the effect of adherence behavior using data from the placebo arms of the WHI. [abstract]. Arthritis Rheum. 2009;60 Suppl 10:613. doi:10.1002/art.25693.

8. Silverman SL, Cramer JA, Sunyecz JA, et al (2007) Women are more persistent with monthly bisphosphonate therapy compared to weekly bisphosphonates: 12 month results from two retrospective databases. Presented at ASBMR Montreal Sept 19, 2007. Abstract W366.

9. Gold DT, Safi W, Trinh H. Patient preference and adherence: comparative US studies between two bisphosphonates, weekly risedronate and monthly ibandronate. Curr Med Res Opin. 2006;22:2383-91.

10. Griswold ME, Localio AR, Mulrow C. Propensity score adjustment with multilevel data: setting your sites on decreasing selection bias. Ann Int Med. 2010;152:393-5.

11. Hamilton B, McCoy K, Taggart H. Tolerability and compliance with risedronate in clinical practice. Osteoporos Int. 2003;14:259-62.

12. Kennedy J, Teleu I, Mackay K. Unfilled prescriptions of Medicare beneficiaries: prevalence, reasons, and types of medicines prescribed. J Manage Care Pharm. 2008;14:553-60.

13. Fischer MA, Stedman MR, Lii J, et al. Primary medication nonadherence analysis: analysis of 195, 930 electronic prescriptions. J Gen Intern Med. 2010;25(4):284-90. doi:10.1007/s11606010-1253-9.

14. Boston Consulting Group (BCG) (2002) Analysis: Harris Interactive 10,000 Patient Survey 2002 at http://www.bcg.com/documents/ file14265.pdf accessed October 21,2009.

15. Rees, G., Leong, O., Crowston, J. C. Intentional and Unintentional Nonadherence to Ocular or Hypertensive Treatment in Patients with Glaucoma, Ophthalmology, 2010, doi:10.1016/j.ophtha.2009.10.038.

16. Clifford S, Barber N, Home R. Understanding different beliefs held by adherers, unintentional non-adherers and intentional nonadherers, applications and necessity-concerns framework. J Psychosom Res. 2008;64:41-6.

17. Christensen AJ, Howren MB, Hillis SL, Kaboli P, Carter BL, Cvengros JA, Wallston KA, Rosenthal GE. Patient and physician beliefs about control over health: association of symmetrical beliefs with medication regimen adherence. J Gen Intern Med. 2010;25(5):397-402.
18. Gusdal AK, Obua C, Andaulem T, et al. Voices on adherence to ART in Ethiopia and Uganda: a matter of choice or simply not an option. AIDS Care. 2009;21:1381-7.

19. Trinacty CM, Adams AS, Soumerai SB, et al. Racial differences in long term adherence to oral antidiabetic drug theraphy: a longitudinal cohort study. BMC Health Services Research 2009;9:24-34. doi:10.1186/1472-6963-9-24.

20. Cramer JA. Systematic review of adherence with medicines for diabetes. Diabetes Care. 2004;2712:18-24.

21. Van den Bemt BJ, van den Hoogen FH, Benraad B, et al. Adherence rates and associations with nonadherence in patients with rheumatoid arthritis using disease modifying antirheumatic drugs. J Rheumatol. 2009;36:2164-70.

22. Zivin K, Ganoczy D, Pfeiffer PN, Miller EM, Valenstein M. Antidepressant adherence after psychiatric hospitalization among VA patients with depression. Admin Pol Ment Health. 2009;36 (6):406-15.

23. Cizza G, Primma S, Csako G. Depression as a risk factor for osteoporosis. Trends Endocrinol Metabol. 2009;20(8):367-73.

24. Buist DS, LaCroix AZ, Black DM, Harris F, Blank J, Ensrud K, et al. Inclusion of older women in randomized clinical trials: factors associated with taking study medication in the fracture intervention trial. J Am Geriatr Soc. 2000;48(9):1126-31.

25. Bazargan JKH, Bing EG M. Alcohol consumption and compliance among inner-city minority patients with type 2 diabetes mellitus. Arch Fam Med. 2000;9(10):964-70.

26. Bryson $\mathrm{CL}, \mathrm{Au} \mathrm{DH}$, et al. Alcohol screening scores and medication non-adherence. Ann Intern Med. 2008;149:795-804.

27. Thiebaud P, Patel BV, Nichol MB, Berenbeim DM. The effect of switching on compliance and persistence: the case of statin treatment. Am J Manag Care. 2005;11:670-74.

28. Haynes RB, Ackloo E, Sahota N, McDonald HP, Yao X. Interventions for enhancing medication adherence. Cochrane Database Syst Rev. 2008;16(2):CD000011.

29. Conn VS, Hafdahl AR, Cooper PS, Ruppar TM, Mehr DR, Russell CL. Interventions to improve medication adherence among older adults: meta-analysis of adherence outcomes among randomized controlled trials. Gerontologist. 2009;49(4):447-62.

30. Russell CL. A clinical nurse specialist-led intervention to enhance medication adherence using the plan-do-check-act cycle for continuous self-improvement. Clin Nurse Spec. 2010;24(2):69-75.

31. Schackman BR, Dastin Z, Rubin DS. Feasibility of using audio computer assisted self interview (ACASI) screening in routine HIV care. AIDS Care. 2009;21:992-9.

32. Wilson Sr, Stroub P, Buist AS, et al. Shared treatment decision making improves adherence and outcomes in poorly controlled asthma. Am J Respir Crit Care Med. 2010;181:566-77. 